\title{
Dispotismo. Genesi e sviluppi di un concetto filosofico-politico, a cura di Domenico Felice
} Napoli, Liguori 2002 («Memo», 3), 2 vols

\section{Stefano B. Galli}

\section{(2) OpenEdition}

\section{Journals}

\section{Edizione digitale}

URL: http://journals.openedition.org/studifrancesi/32716

DOI: $10.4000 /$ studifrancesi.32716

ISSN: 2421-5856

\section{Editore}

Rosenberg \& Sellier

\section{Edizione cartacea}

Data di pubblicazione: 1 décembre 2005

Paginazione: 601-603

ISSN: 0039-2944

\section{Notizia bibliografica digitale}

Stefano B. Galli, «Dispotismo. Genesi e sviluppi di un concetto filosofico-politico, a cura di Domenico Felice», Studi Francesi [Online], 147 (XLX | III) | 2005, online dal 01 novembre 2015, consultato il 19 avril 2021. URL: http://journals.openedition.org/studifrancesi/32716; DOI: https://doi.org/10.4000/ studifrancesi.32716

\section{Questo documento è stato generato automaticamente il 19 avril 2021.}

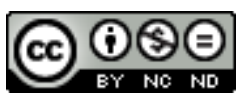

Studi Francesi è distribuita con Licenza Creative Commons Attribuzione - Non commerciale - Non opere derivate 4.0 Internazionale. 


\title{
Dispotismo. Genesi e sviluppi di un concetto filosofico-politico, a cura di Domenico Felice
}

\author{
Napoli, Liguori 2002 («Memo», 3), 2 vols
}

\author{
Stefano B. Galli
}

\section{NOTIZIA}

Dispotismo. Genesi e sviluppi di un concetto filosofico-politico, a cura di Domenico Felice, Napoli, Liguori 2002 («Memo», 3), 2 vols, XVII-701 pp.

1 Studiare la politica significa anzitutto analizzare, sotto il profilo etimologico e anche filologico, il linguaggio attraverso il quale essa si esprime; un linguaggio che ha smarrito il significato originario del termine dispotismo, oggi generalmente adottato per identificare qualsiasi forma di governo ab-solutus - dalla tirannìa alla dittatura, dall'autocrazia all'assolutismo - dalla quale esso, tuttavia, si differenzia radicalmente. Il dispotismo, infatti, è quella forma di governo nell'ambito della quale il detentore del potere ha nei confronti dei suoi governati il medesimo rapporto che il padrone (il despot\%os) intrattiene con i propri schiavi. È proprio qui, relativamente al vincolo di subordinazione - non già nell'assenza del limite della legge (il princeps legibus solutus) e al rapporto del despota con i sudditi, che si misura la differenza del dispotismo rispetto alle forme di governo alle quali troppo spesso, e superficialmente, tale concetto viene assimilato. Si tratta di un concetto che illustra il volto «demoniaco» del potere e appartiene tout court al vocabolario della filosofia e della teoria politica e giuridica e che tiene insieme gli apparati speculativi dei grandi pensatori nel ciclo di lunghissimo periodo che va dall'antichità classica sino alle soglie del secondo Millennio. Una manifestazione del potere che ha catturato le attenzioni scientifiche anche del compianto Norberto Bobbio, autore della bella voce «Dispotismo» che appare nel noto 
Dizionario di politica diretto insieme a Nicola Matteucci e a Gianfranco Pasquino (Torino, Utet, 1983).

2 È dunque da accogliere positivamente la pubblicazione di questo ampio, articolato e approfondito contributo, che presentiamo con imperdonabile ritardo, dedicato, appunto, al tema del dispotismo e curato da Domenico Felice, studioso noto per i suoi costanti e ricorrenti approfondimenti della filosofia politica montesquieuiana (è membro del Consiglio scientifico della Société Montesquieu e ha pubblicato, tra l'altro: Pour l'histoire de la fortune de Montes-quieu en Italie, Bologna 1990, che è stato insignito del «Prix Montesquieu 1991»; Modération et justice. Lectures de Montesquieu en Italie, Bologna 1995 e Oppressione e libertà. Filosofia e anatomia del dispotismo nel pensiero di Montes-quieu, Pisa 2000). Quel che colpisce scorrendo i nomi e, soprattutto, le biografie - riportate nelle ultime pagine - degli autori degli interventi in questo volume collettaneo in due tomi dedicato al concetto del dispotismo e alla sua evoluzione storica nell'ambito della filosofia e della speculazione teorico-politica, è la loro giovane età.

Questo dato attribuisce al volume una "freschezza» che, sotto la consumata regìa di Domenico Felice, s'è tradotta in una agilità di scrittura e in una originalità interpretativa davvero interessanti e suggestive in quasi tutti i saggi proposti. Oltre a studiosi di consolidata esperienza e comprovato spessore come Giorgio Bongiovanni su Hegel insieme ad Antonino Rotolo, Pietro Capitani sui Fisiocrati e Mably, come il compianto Anselmo Cassani e Nadia Urbinati su John Stuart Mill, ma anche come Giovanni Cristani su Boulanger e Edoardo Greblo su Rousseau, Manlio Iofrida su Wittfogel, Margherita Isnardi Parente su Bodin e lo stesso Felice quasi «ovviamente» su Montesquieu, tra gli autori troviamo infatti i contributi - e solo per fare alcuni esempi di Thomas Casadei sul dispotismo nel pensiero di Hannah Arendt, di Cristina Cassina su Tocqueville, oppure ancora di Alessandro Ceccarelli su Turgot e Condorcet, di Claudio Fiocchi e Stefano Simonetta sull'aristotelismo politico bassomedievale, di Marina Lalatta Costerbosa su Locke, di Paola Mittica e Silvia Vida su Aristotele, di Giovanni Paoletti su Constant, di Cristina Passetti sul pensiero dei giacobini, di Viola Recchia su Helvétius, di Giorgio Scichilone su Machiavelli. Da questa rapida rassegna degli autori e dei loro saggi è possibile intuire l'impianto del lavoro sul dispotismo coordinato da Domenico Felice. Seguendo un rigoroso approccio deliberatamente confinato nell'ambito della filologia $\mathrm{e}$, dunque, della fedeltà al testo scritto più che alle sue interpretazioni, questi studiosi affrontano e analizzano le varie concettualizzazioni del dispotismo secondo prospettive che spaziano dalla speculazione filosofica e politicodottrinaria alla riflessione antropologica e giuridico-istituzionale e ne ricostruiscono la storia, da Aristotele alla Arendt.

4 Senza dubbio, la riflessione sul dispotismo, sin dagli albori del ciclo storico della modernità politica, costituisce un elemento essenziale delle analisi sull'origine e sulla fisionomia del potere e sulle sue manifestazioni concrete. Si tratta di una riflessione che trova nella cultura politica e giuridica francese tra il XVI e il XIX secolo il suo nucleo più denso e forse anche più maturo e che - da Bodin all'assolutismo dispotico del Re Sole, da Montesquieu a Boulanger, da Helvétius a Rousseau, dai Fisiocrati a Mably, da Voltaire a Linguet, da Turgot a Condorcet, da Marat a Robespierre, da Constant a Tocqueville - si configura come un'autentica polifonia di voci nell'ambito della quale giganteggia la figura del Presidente del parlamento di Bordeaux. Nella genesi e nello sviluppo del concetto di dispotismo, Montesquieu - che nacque nel cuore del dérapage dispotico di Luigi XIV e visse con matura consapevolezza la successiva 
degenerazione della Reggenza e del sistema dei Consigli, proprio nel momento in cui, oltre la Manica, trionfava il superamento parlamentare dell'assolutismo - rappresenta davvero una pietra angolare. Prima della riflessione montesquieuiana, il dispotismo veniva considerato come uno dei «volti» della monarchia, anzi una vera e propria degenerazione della sua forma "assoluta», alla quale era indissolubilmente legato. Per la prima volta in modo organico e sistematico sotto il profilo dottrinario, con Montesquieu - come osserva Felice nelle prime pagine del suo saggio - il dispotismo diventa «un genere di governo distinto o a sé stante, alla stessa stregua del genere repubblica e del genere monarchia» (t. I, pp. 190-191; i corsivi sono nel testo). Insomma, per citare ancora Felice, «laddove gli uni 'abbassano' o 'degradano', per così dire, lo Stato dispotico al rango di sottospecie della specie monarchia, Montesquieu lo 'innalza' o 'eleva' alla dignità di tipo primario o fondamentale di governo, conferendogli un rilievo $\mathrm{e}$ un ruolo davvero eccezionali, quali mai s'erano visti fino ad allora e che si ritroveranno successivamente solo nelle Lezioni sulla filosofia della storia di Hegel» (ibidem; i corsivi sono nel testo).

Effettivamente, l'innovazione montesquieuiana nella interpretazione del dispotismo, riferito agli antichi Stati orientali, è rilevante, determina una vera e propria svolta nel quadro della teoria politica e conferma - quasi ve ne fosse il bisogno - la levatura e l'importanza dell'autore dello Spirito delle leggi nella fondazione delle scienze sociali e nella sistematizzazione dell'analisi dei sistemi politici e istituzionali; analisi così elevata al rango di vera e propria «scienza», al pari di quelle esatte. Proprio per questo, la teoria dei governi elaborata dal Presidente del parlamento di Bordeaux va oltre la tradizionale tripartizione platonica e, soprattutto, aristotelica (monarchia, aristocrazia e democrazia e loro degenerazioni), e approda a una sistematizzazione di tipo qualitativo - non già quantitativo - sia in ordine al numero di coloro o colui che gestisce il potere, sia relativamente ai metodi che vengono adottati nell'ambito del suo esercizio. Più che nel quadro delle scienze giuridiche, la speculazione montesquieuiana si colloca deliberatamente, pertanto, nell'alveo delle dottrine politiche e sociologiche, poiché essa tende a individuare quelle forze che «abitano» le istituzioni dello Stato e così ad attribuire a esse una precisa fisionomia.

6 Dispotismo e libertà rappresentano una dicotomia davvero inscindibile nella sua struttura teorica; e tuttavia, nel corso degli anni rivoluzionari si verificò un autentico «dispotismo della libertà», che per certi aspetti ribaltò e mise in discussione il nesso tra il dispotismo e la generalizzata negazione di libertà politica e civile che lo caratterizzava. Come osserva Cristina Passetti nel suo saggio, sin dal 1789 «nello svelare al mondo che la libertà politica poteva essere conquistata da tutti i popoli, perché non legata ad alcun carattere nazionale, la Rivoluzione francese presentò la lotta universale dei popoli contro le teste coronate come lotta della libertà contro il dispotismo» (t. II, p. 420 ; il corsivo è nel testo). Effettivamente, quella rivoluzionaria fu una vera e propria lotta senza quartiere per la libertà e contro il dispotismo che nella successiva degenerazione giacobina - definita giustamente e prudentemente da Passetti, sin dal titolo del suo saggio, «eccezione» - si configurò come la coniugazione di due concetti politici tra loro antitetici. «Dispotismo della libertà»: sotto il profilo del vocabolario politico questa espressione indicava una visione del mondo che si definiva attraverso il ricorso ad un lessico proprio della cultura dell'Ancien régime e tuttavia carico di nuovi significati fortemente ideologizzati. 
7 Con l'eredità della cultura politica rivoluzionaria dovettero fare i conti i due grandi teorici del costituzionalismo moderno e della democrazia liberale: Benjamin Constant e Alexis de Tocqueville. Il primo cercò di riflettere sulle nuove sembianze assunte dal dispotismo nel primo quarto del XIX secolo. Il «dispotismo dei moderni» rappresenta l'indicatore privilegiato dell'operazione compiuta da Constant di applicare i principi del liberalismo al mutamento storico, pena l'esclusione dalla storia, e dunque il tentativo di analizzare teoricamente le forme nuove di oppressione politica e il lascito del dérapage rivoluzionario successivo alla fase costituente e monarchico-costituzionale nelle sue pratiche restrittive della libertà individuale, politica e civile. Anche lo studioso normanno concentrò la propria attenzione sul dispotismo di «nuova specie» del quale peraltro individuò -secondo un'interpretazione per così dire «estensiva»e, comunque, complessa - quattro volti strettamente connessi all'ineludibile processo di progressiva affermazione della democrazia: il dispotismo assembleare legislativo, la tirannide della pubblica opinione, il cesarismo e lo Stato-Leviatano burocratico e accentratore. Si tratta di forme dispotiche indubbiamente meno violente rispetto a quelle del passato, eppure non per questo meno insidiose ed efficaci dal punto di vista dell'equilibrio e della stabilità del nuovo ordine politico democratico, soprattutto nei suoi rapporti con la libertà individuale e collettiva.

8 Nel Novecento la riflessione sul fenomeno dispotico ha fortemente risentito della presenza del totalitarismo, soprattutto con la speculazione di Karl Wittfogel, che riprende il «mito» del dispotismo orientale, e di Hannah Arendt, sensibile e attenta all'evoluzione della forma demoniaca del potere e alle sue capacità di inghiottire le libertà individuali. Wittfogel ne enfatizza l'aspetto realistico, in termini storici, e si pone in linea di continuità rispetto alla tradizionale trattazione del dispotismo, analizzando il carattere assoluto di tale forma di potere, la paura diffusa, il vincolo di subordinazione unilaterale, la durata nel tempo, i suoi rapporti con la teocrazia. In questo quadro, egli inserisce il ruolo dei potenti apparati burocratici degli Stati orientali (il dispotismo «idraulico»), che incidono sulla produttività agricola, e dunque regolamentano sotto il profilo tecnico i rapporti dell'uomo con il suolo, al di là della natura dei singoli popoli.

Come osserva Thomas Casadei nelle prime righe del suo saggio su Hannah Arendt, il fenomeno totalitario è stato talvolta interpretato quale prerogativa specifica del XX secolo, differente da precedenti tipologie statuali, tal altra come l'ultima manifestazione di antiche e consolidate forme autoritarie e tiranniche del passato. Scrive Casadei: «Se la realtà del totalitarismo, come evidenzia la Arendt, oltre a essere una realtà complessa, intricata, molteplice e plurale, è soprattutto, per qualità e dimensioni, nuova, mai esperita prima, pare altresì possibile rinvenire nell'amalgama degli elementi che la compongono certe cospicue affinità con alcuni tratti strutturali già presenti, anche solo in nuce, nel dispotismo [...]» (t. II, pp. 627-628). Si tratta allora di un'autentica metamorfosi: il dispotismo si è trasfigurato ed è riapparso come forma demoniaca del potere, sotto mutate sembianze, come totalitarismo ed ha dato un volto a quel Novecento che abbiamo appena archiviato alle nostre spalle e che in esso ha trovato una delle sue plurime, e deteriori, identità politiche e ideologiche. 